\title{
Overview of Direct Detection Dark Matter Experiments
}

\section{Jodi Cooley}

Southern Methodist University

Dallas, TX, USA

E-mail: cooley@physics.smu.edu

The past year has yielded several new results from the direct detection dark matter community. In this paper, I will give a review of the techniques used by experimentalists for the direct detection of dark matter and I will show the most recent results from several experiments in the field and report their current status.

VIII International Workshop on the Dark Side of the Universe

Rio de Janeiro, Brazil

June 10-15, 2012 


\section{Introduction}

The evidence for the existence of dark matter largely comes from its gravitational effects. First postulated by Fritz Zwicky nearly 80 years ago [1]. However, it was studies of the rotation curves of galaxies in the 1970s and early 1980s by Whitehurst and Roberts [2], Rubin et. al [3] and Bosma [4] that made it clear that this nonluminous matter must exist. Furthermore, the revolution in precision cosmology of the last decade has revealed conclusively that about a quarter of our universe consists of dark matter [5]. Despite the abundant evidence for the existence of dark matter, its constituents have eluded detection.

Phenomenology at the intersection of particle physics, cosmology and astrophysics gives well-motivated candidates for dark matter. Dark matter candidates naturally arise from theories that explain the radiative stability of the weak scale. These theories include supersymmetry [6] and theories of extra dimensions [7, 8, 9]. Candidates from these theories are representative of a generic class of weakly interacting massive particles (WIMPs). In the hot early universe WIMPs would have been in thermal equilibrium. They would have decoupled as the universe expanded and cooled, leaving behind a "relic abundance" of these particles in the universe today. In order to explain the observed relic abundance, the WIMP interaction cross section must be consistent with the weak scale.

If dark matter is composed of WIMPs, their existence in the local galactic halo could be detected through their elastic scattering off nuclei in a terrestrial detector. The expected interaction rate is quite low, less than 0.01 event $/ \mathrm{kg}$-day [10], much lower than the radioactive background of most materials. Hence, such a detector would need to be housed deep underground to protect it from cosmic rays and fabricated using radioactivity-free detector materials.

\section{Detection Techniques}

The current experiments employ one of two strategies for detecting dark matter. The first strategy is to look for an annual modulation of WIMPs due to the earth's rotation around the sun as the sun travels through the solar system. The difficulty with this method is that one looks for a small, time-varying signal on top of a huge background. It requires a large detector volume and a stable, nonfluctuating background. The advantage is one does not need to know the characteristics of the background as long as it is not time varying.

The other strategy is to substantially reduce the background to near zero and detect WIMPnucleon interactions. The difficulty with this approach is characterizing the backgrounds and achieving an extremely low-background experimental environment.

Globally there are a variety of detector mediums used including crystals such as $\mathrm{Ge}, \mathrm{Si}$, CaWO4 and liquid noble gasses such as Xe and Ar. There are three observables from the interactions of WIMPs and the target medium: ionization, phonons resulting from the interaction of the WIMP with a nucleon in the crystal lattice, and scintillation. Different experiments use one or two of these observables to differentiate candidate signal events from the ubiquitous background. 
Dark matter candidates and neutrons are assumed to interact with the target's nucleus producing nuclear recoils while most background particles ( $\gamma$-rays and electrons) interact with electrons surrounding the nucleus producing electron recoils. Experiments that use two observables have excellent discrimination between candidate signal events and backgrounds can be obtained from the fractional energy (i.e. ionization energy divided by phonon energy). This quantity is known as "yield".

\section{Backgrounds}

Dark matter experiments are faced with a variety of background sources including products of cosmic ray interactions and radioactivity from the environment and detector materials. Although the specifics of dealing with these backgrounds vary from one experiment to another, the general techniques used to reduce these sources are the same.

Dark matter detectors are sited in deep underground laboratories in order to reduce the number of neutrons produced by cosmic ray muon interactions. As an illustration of the effect that substantial depth can have consider the neutron rate from cosmic rays at 15 meters water equivalent (mwe) is approximately $1 /(\mathrm{kg} \mathrm{s})$. However, at 2000 mwe that rate reduces to approximately $1 /(\mathrm{kg}$ day). Another technique employed by dark matter experiments to reject these neutrons is to surround the detectors with active muon vetoes made of either scintillating panels or water shields which can be used to reject events from muon interactions.

Neutrons from natural radioactivity are another background that dark matter experiments frequently encounter. These neutrons result from $(\alpha, n)$ reactions and spontaneous fission of uranium and thorium nuclides that are natural in all geological formations. An $(\alpha, n)$ reaction occurs when uranium or thorium decays giving off an $\alpha$-particle which can react with elements such as $\mathrm{Al}$ or Na producing a neutron. The number and energy of neutrons produced from these reactions varies depending on the type of rock. A passive shielding of hydrocarbons such as polyethylene is typically used to reduce these backgrounds.

Finally, photons, which can come from environmental radioactivity or radiative contaminants in the detector itself or its shielding, may also contribute to the background seen by dark matter detectors. To reduce this type of background experiments frequently use passive shielding such as ancient lead and copper. In an effort to further reduce the photon background, the detectors and shielding are built using low-radioactivity materials.

\section{Experimental Results}

Dark matter experiments can be divided into two categories: those that observe an excess of events over their predicted backgrounds and those that do not see an excess of events over their predicted backgrounds. The most pressing task is to understand the seemingly contradictory results seen by these experiments through further experimentation and understanding of physics required to interpret the results. The following subsections will review and highlight recent results from leading experiments. 


\subsection{CRESST II}

The CRESST II experiment [11] is located in the Laboratroi Nazionali de Gran Sasso, Italy at a depth of approximately 3500 mwe. The detector consists of eight $\mathrm{CaWO}_{4}$ crystals instrumented to readout phonon energy and scintillation. Each crystal has a mass of $\sim 300 \mathrm{~g}$ and is operated at a temperature of $\sim 10 \mathrm{mK}$. Discrimination between electron recoil and nuclear recoil events comes from light yield, the ratio of scintillation energy to phonon energy. The dominant background from radioactivity produces electron recoils.

The most recent results are obtained from a net exposure of $730 \mathrm{~kg}$-day (June 2009 March 2011). In that time 67 events were observed in the acceptance region. The analysis used a maximum likelihood in which two regions favored a WIMP signal in addition to known backgrounds.

In the next data run the CRESST II collaboration aims to increase the mass of their detector and reduce the considerable number of background events. A new clamp design is anticipated to reduce the number of alpha events and additional shielding is expected to reduce the overall number of events.

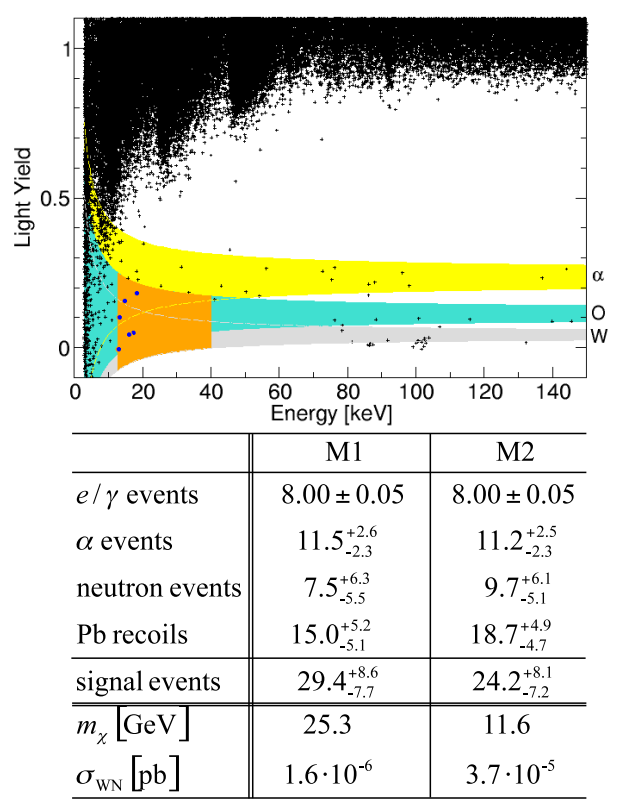

Figure 1: Top: Light yield versus energy of events seen in one module during $730 \mathrm{~kg}$-days of exposure. In this module six events appear in the acceptance region (orange). Also shown are the (90\%) bands for the $\alpha$ (yellow) oxygen (cyan) and tungsten (grey) nuclear recoils. Bottom: Expected backgrounds and possible WIMP signal resulting from the maximum likelihood analysis [8].

\subsection{DAMA/LIBRA}

The DAMA/LIBRA experiment is located in the Laboratroi Nazionali de Gran Sasso, Italy at a depth of approximately 3500 mwe. The DAMA experiment was an $100 \mathrm{~kg}$ array of scintillating $\mathrm{NaI}$ crystals operated from 1996 to 2002. The LIBRA experiment is an array of $250 \mathrm{~kg}$ scintillating NaI crystals from which first results were reported in 2008. 
The DAMA/LIBRA experiment does not distinguish between a WIMP signal and background events directly. Instead it looks for a signal from the amplitude of the annual modulation of the WIMPs due to Earth's rotation around the sun as our solar system travels through the WIMP halo of our galaxy.

The DAMA/LIBRA collaboration has observed a modulation of events seen in their experiment consistent of that expected from the WIMP halo for over 13 cycles. The signal is only seen in the experiment's lowest energy bin. However, its significance is $8.9 \sigma$.

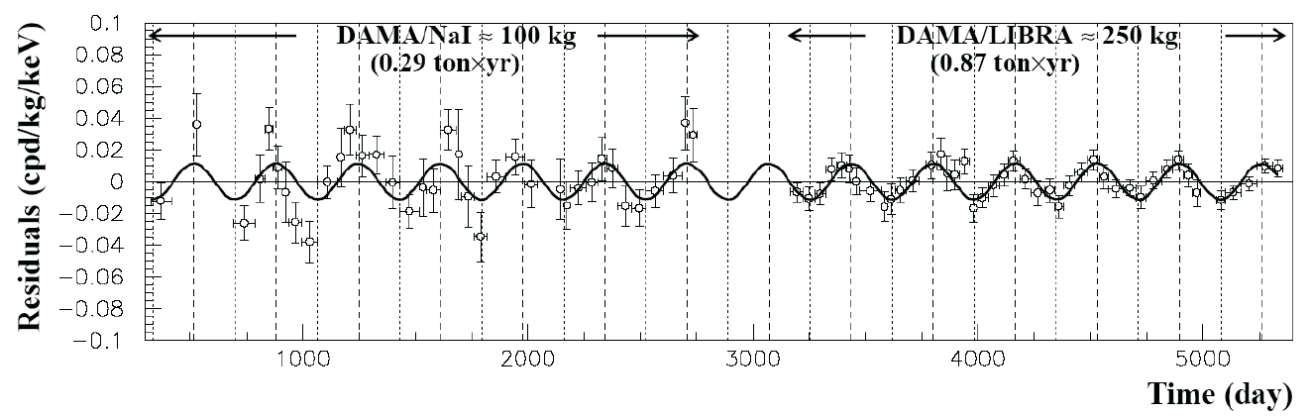

Figure 2: Residual rate of candidate WIMP events measured by the DAMA/LIBRA experiments. The solid black line is the best fit of the cosinusoidal function with a period of 1 year and phase of 152.5 days during 1.17 ton-years of exposure [12].

\subsection{CoGeNT}

The CoGeNT experiment is located in the Soudan Underground Laboratory, Minnesota, USA at a depth of 2090 mwe. The CoGeNT experiment consists of a 440 g P-type Point Contact (PPC) detector that acts as an ionization spectrometer. This experiment reports an exponential excess of events at low energies based on 442 live-days spanning the period from December 4, 2010 to March 6, 2011 after subtracting off known backgrounds due to cosmogenic activation of the germanium detector as shown in Fig. 3.

The CoGeNT collaboration performed a modulation analysis [13] of these events. In the energy range $0.5-3.0 \mathrm{keV}_{\text {ee }}$ they report a modulation $2.8 \sigma$ C.L. The favored modulation has an amplitude of $16.6 \pm 3.8 \%$, period of $347 \pm 29$ days, with a minimum on Oct. $16 \pm 12$ days.

\subsection{CDMS II and SuperCDMS}

The CDMS II experiment was located in the Soudan Underground Laboratory, Minnesota, USA at a depth of 2090 mwe. The CDMS II experiment consisted of $30 \mathrm{Z}$-sensitive Ionization and Phonon (ZIP) detectors (19 Ge and $11 \mathrm{Si}$ ) which were installed and operated from June 2006 to March 2009. The detectors were photolithographically patterned with Al fins and W transition edge sensors (TESs) to collect athermal phonons and ionization energy.

Discrimination between electron recoil and nuclear recoil events comes from the simultaneous measurement of phonon and ionization energy. Ionization yield is defined as the ratio of ionization energy to phonon energy. Electron recoils have an ionization yield of $\sim 1$, while nuclear recoils have an ionization yield of $\sim 0.3$. 
Electrons interacting near the detector surface often suffer from a reduced ionization yield due to charge-carrier back-diffusion and can mimic the nuclear recoil signature. These events produce a different frequency of phonons that result in shorter rise-times of the phonon pulse and can be distinguished by parameterizing the timing characteristics of each event.

Results from the full data taken by the CDMS II yielded an upper limit of $3.9 \times 10^{-44} \mathrm{~cm}^{2}$ for WIMPs of mass $70 \mathrm{GeV} / \mathrm{c}^{2}$. at the $90 \%$ C.L. on the WIMP-nucleon cross-section [14].

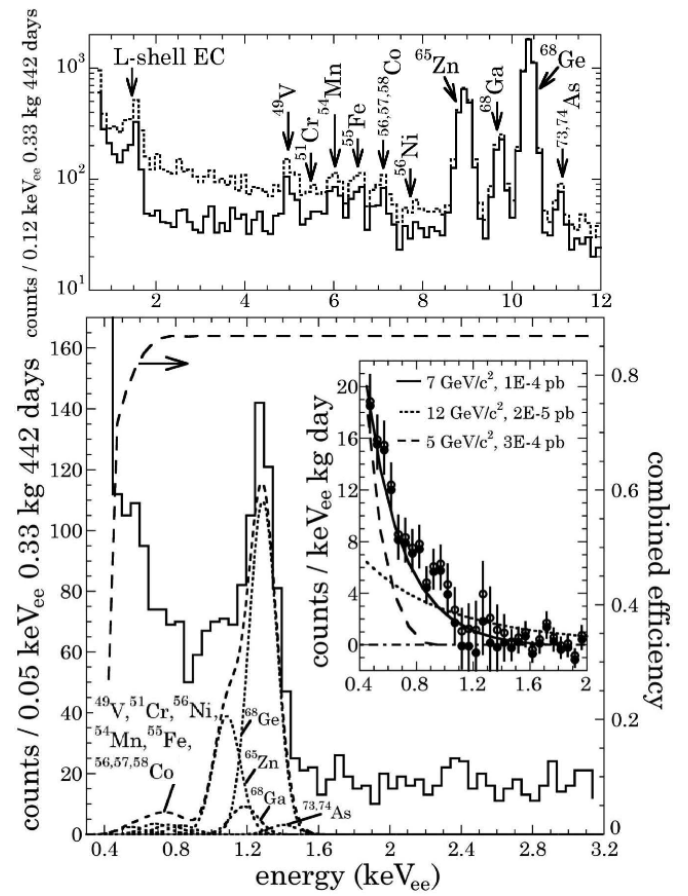

Figure 3: Top: Spectrum of expected peaks prior to threshold energy correction from K-shell electron capture and cosmogenic peaks before (dotted) and after (solid) surface events are removed. Bottom: Low-energy spectrum after the removal of surface events. The dotted line with arrow indicates the trigger threshold efficiency. Inset: Spectra after L-shell contributions and threshold efficiency are applied overlapped with example light WIMP signals [13].

The CDMS II collaboration has recently performed a reanalysis of the CDMS II data using a lower energy threshold of $2 \mathrm{keV}$ in order to increase the sensitivity to WIMPs lighter than 10 $\mathrm{GeV} / \mathrm{c}^{2}$. This recent analysis used the data taken between October 2006 and September 2008 from the 8 detectors with the lowest trigger thresholds $(1.5-2 \mathrm{keV})$. This resulted in $241 \mathrm{~kg}-$ days of livetime. No phonon timing cut to distinguish surface events was used as it is ineffective below $5 \mathrm{keV}$.

Assuming all events were WIMP candidates, an upper limit was set using the Yellin Optimal Interval method. The limit produced at the 90\% C.L. level is incompatible with an interpretation of spin-independent inelastic WIMP-nucleon scattering of the excess events seen by DAMA/LIBRA and CoGeNT [15]. These results are shown in Fig. 4.

In addition, the CDMS II collaboration preformed a modulation analysis of events from the reanalysis using the lower energy threshold. The energy range considered for this analysis was $5.0-11.9 \mathrm{keV}_{\mathrm{nr}}$, which maps to the $1.2-3.2 \mathrm{keV}_{\mathrm{ee}}$ equivalent recoil energy interval in the 
CoGeNT experiment. This analysis rules out modulated rates greater than $0.07 \mathrm{keV}_{\mathrm{nr}}[\mathrm{kg} \text {-day }]^{-1}$ at the $99 \%$ C.L. for events in the nuclear recoil band.

The SuperCDMS at Soudan experiment is currently operating 5 towers of advanced iZIP detectors $(\sim 10 \mathrm{~kg} \mathrm{Ge})$ in the existing cryostat at the Soudan Underground Laboratory. After 2 years of operation, the sensitivity of the experiment to WIMP-nucleon interactions is expected to improve by a factor of four over existing CDMS II results.

The new iZIP detector design interleaves phonon and charge sensors on both detector faces. This allows for separation of bulk and surface events from the symmetry of the ionization collection in addition to the traditional yield and pulse shape parameters of the ZIP detector design. Bulk events will have equal but opposite ionization signals appearing on both sides of the detector while surface events will have ionization signal that appears on only one detector face.

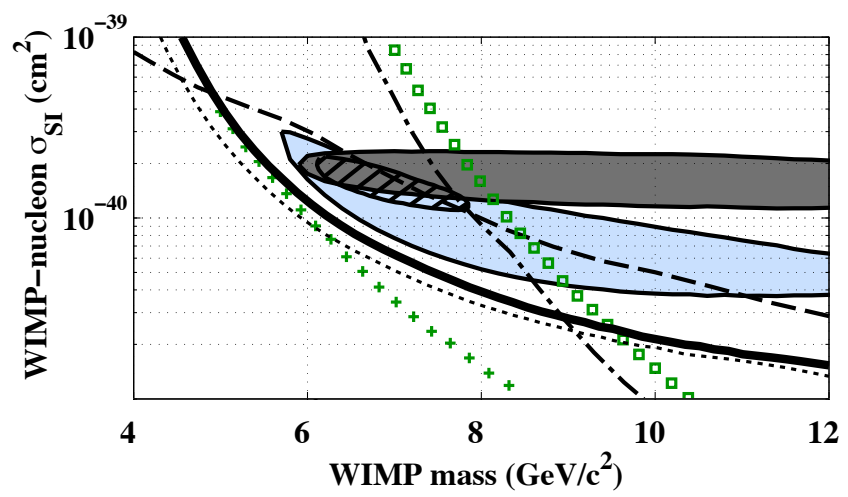

Figure 4: Comparison of the spin-independent WIMP-nucleon cross section exclusion limit from CDMS II reanalysis (solid), CDMS II shallow-site (dotted), CDMS II traditional analysis (dash-dotted), and XENON 100 with constant (pluses) or decreasing (boxes) scintillation efficiency. Filled regions represent DAMA/LIBRA (grey), CoGeNT (light blue), and combined fit to DAMA/LIBRA and CoGeNT (hatched) possible signal regions. Figure taken from [16].

\subsection{EDELWEISS-II}

The EDELWEISS-II experiment was located in the Laboratoire Souterrain de Moudan (LSM) located between Italy and France at a depth of 4800 mwe. Similar to CDMS, EDELWEISS II measures both phonons and ionization. However, instead of using TESs to measure phonon energy, EDELWEISS-II measures heat using a neutron transmutation doped (NTD) Ge thermal sensor.

Current results from EDELWEISS-II are based on $427 \mathrm{~kg}$-days of data obtained from ten $400 \mathrm{~g}$ Ge detectors that were operated from 2008 - 2010. Five events were observed in the nuclear recoil band with an expected background of 3 events. This yielded an upper limit on the spin independent WIMP-nucleon cross section of $4.4 \times 10^{-44} \mathrm{~cm}^{2}$ for a WIMP of mass 85 $\mathrm{GeV} / \mathrm{c}^{2}$ at the $90 \%$ C.L [17]. 
The EDELWEISS Collaboration is in the process of upgrading their detector to EDELWEISS-III. Their goal is to obtain $3000 \mathrm{~kg}$-days by increasing the mass of each of their detectors to $800 \mathrm{~g}$ and using new interdigitated ZIPs which readout charge on both faces of each detector. In addition, they plan to reduce their background by a factor of 10 through increased shielding, material selection and better surface event rejection.

\subsection{XENON}

The XENON experiment is located in the Laboratroi Nazionali de Gran Sasso, Italy at a depth of approximately 3500 mwe. The XENON detection principle uses a two-phased time projection chamber readout by photomultiplier tubes (PMTs) located on the top and bottom of the chamber. The bottom PMTs are immersed in liquid xenon and detect primary scintillation from the interaction of charge particles in the liquid. Electrons from this primary interaction are drifted through an electric field into the gaseous xenon phase where they scintillate. This secondary scintillation is detected by PMTs at the top of the chamber. The ratio between the secondary and primary scintillation provides discrimination between electron and nuclear recoils.

The most recent results from this program are based on 100.9 live days acquired from January through June 2010 from the XENON100 detector. The fiducial mass of the detector was $48 \mathrm{~kg}$ of liquid XENON. Three events were observed with a predicted background of 1.8 \pm 0.6 gamma events and $0.1 \pm 0.08 \pm 0.04$ neutron events yielding an upper limit on the spinindependent WIMP-nucleon cross section of $7.0 \times 10^{-45} \mathrm{~cm}^{2}$ for a WIMP of mass $50 \mathrm{GeV} / \mathrm{c}^{2}$ [18].

The XENON100 experiment continues to take data. In addition, the collaboration is planning for an upgrade to XENON1T [19]. This upgrade consists of a 2.2 ton liquid xenon TPC with 1 ton fiducial mass in a $10 \mathrm{~m}$ water shield. It has been approved by INFN for installation in Gran Sasso. Construction is expected to begin later this year (2012) with first science data to be taken in 2015. The projected sensitivity to spin-independent WIMP-nucleon scatter of XENON1T is $2 \times 10^{-47} \mathrm{~cm}^{2}$ after 2 years of running.

\subsection{Single Phase Liquid Noble Experiments: XMASS and DEAP/CLEAN}

XMASS [20] is a single-phase liquid xenon detector surrounded by a water tank that acts as a muon veto. The experiment is located in the Kamioka Underground Observatory, Japan at a depth of 2700 mwe. Construction of the experiment finished in late 2010. The key concept to background discrimination is "self-shielding", whereby gamma particles are absorbed in the outer region of the liquid xenon and do not reach the innermost fiducial volume. WIMPs and neutrons would be evenly distributed throughout the volume. Hence, particles reaching the inner most region of the detector would have a high probability of being either WIMPs or neutrons. The most recent science run of the XMASS detector revealed an unexpected alpha particle background. The collaboration is working to overcome this problem. 


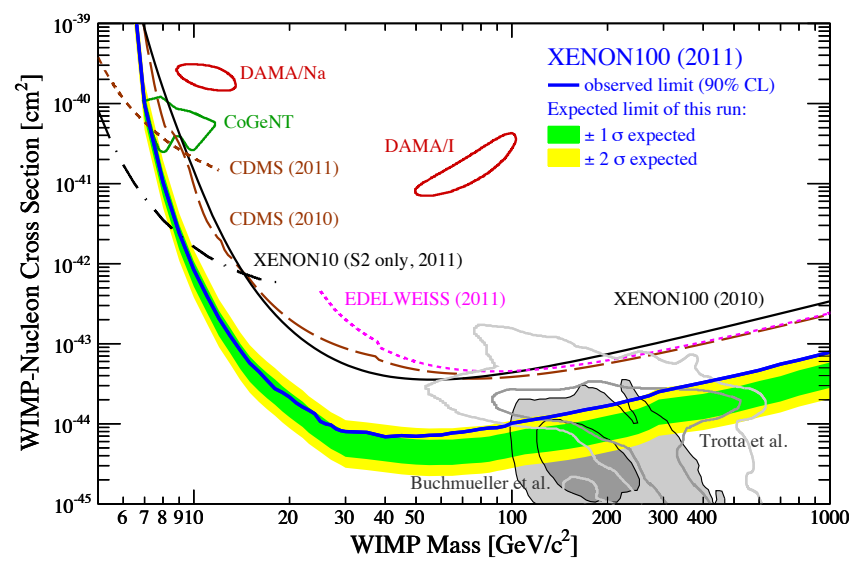

Figure 5: Comparison of the spin-independent WIMP-nucleon cross section exclusion limit from XENON100 (blue, solid), CDMS II traditional analysis (brown dashed), CDMS II low mass (brown dotted), EDELWEISS (pink, dotted) and XENON10 (black, dash-dotted). Also shown are DAMA (red) and CoGeNT (green) favored WIMP signal regions [18].

The DEAP/CLEAN collaboration has a phased program using single-phase liquid argon detectors. The miniCLEAN [21] detector is $150 \mathrm{~kg}$ in fiducial volume with construction anticipated to finish this year (2012) and science run following. It anticipates a spinindependent WIMP-nucleon cross-section sensitivities of $\sim 2 \times 10^{-45} \mathrm{~cm}^{2}$. DEAP 3600 [22] is a one ton fiducial volume detector under construction since 2010. The first science run is anticipated to start in 2013. Its anticipated sensitivity to the spin-independent WIMP-nucleon cross section is of $\sim 1 \times 10^{-45} \mathrm{~cm}^{2}$. The final phase is DEAP/CLEAN, a 10 ton fiducial volume experiment with anticipated sensitivity to the spin-independent WIMP-nucleon cross section of $\sim 1 \times 10^{-47} \mathrm{~cm}^{2}$.

Discrimination in the DEAP/CLEAN experiments comes from differences in pulse shapes between electron recoils and nuclear recoils. The difference comes from the way excited atoms decay to their ground state through the formation of single or triplet excimer states. These states have different decay times. Seventy percent of excimer states created by nuclear recoils are singlets while $30 \%$ of excimer states created by electron recoils are triplets. A parameterization of the prompt emission from these decays provides a reliable discriminator between electron recoils and nuclear recoils.

\section{Summary and Outlook}

The direct detection dark matter field is currently undergoing a surge in activity. There are a number of experiments using a variety of techniques, which I did not have time to talk about. Furthermore, there are plans to extend these existing experiments and techniques to even larger detectors in the next decade. Many experiments have reported new results over the last year. Three of these experiments have reported an observation of an excess of events over their predicted background eluding to a WIMP-signal, while others with sensitivities to the same phase-space have excluded the interpretation of these events as a WIMP signal.

This is truly an exciting and very active time in the field of direct detection dark matter 
physics. Over the course of the next two years, we anticipate the announcement of more results from experiments that have increased sensitivity that will help to shed light on recent observations.

\section{References}

[1] F. Zwicky, Spectral Displacement of Extra Galactic Nebulae, Helv. Phys. Acta. 6 (110) 1933.

[2] Roberts and Whitehurst, Astrophys. J. 201 (327) 1974

[3] V. C. Rubin, et al., Astrophys. J., 238 (471) 1980

[4] A. Bosma, Astron. J. 86 (1825) 1981

[5] E. Komatsu et al., Five-Year Wilkinson Microwave Anisotropy Probe (WMAP Observations: Cosmological Interpretation, Astrophys. J. Suppl., 180 (330) 2009.

[6] G. Jungman, M. Kamionkowski, and K. Griest, Supersymmetric dark matter, Phys. Rept. 267 (195) 1996 [hep-ph/9506380]

[7] K. Agashe and G. Servant, Warped unification, proton stability and dark matter, Phys. Rev. Lett., 93 (231805) 2004 [hep-ph/0403143]

[8] H.-C. Cheng, J. L. Feng, and K. T. Matchev, Kaluza-Klein dark matter, Phys. Rev. Lett. 89 (211301) 2002 [hep-ph/0207125]

[9] G. Servant and T. M. P. Tait, Elastic scattering and direct detection of Kaluza-Klein dark matter, New J. Phys. 4, (99) 2002 [hep-ph/0209262]

[10]J. Engel, S. Pittel, and P. Vogel, Nuclear physics of dark matter detection, Int. J. Mod. Phys. E1 (1), 1992

[11]G. Angloher, et.al, Results from $730 \mathrm{~kg}$ Days of the CRESST-II Dark Matter Search, [arXiv:1109.0702]

[12]R. Bernabei, et. al., New Results from DAMA/LIBRA, in proceedings from Beyond 2010 [arXiv:1002.1028]

[13] C.E. Aalsethm et. al, Search for an Annual Modulation in a P-type Point Contact Germanium Dark Matter Detector, Phys. Rev. Lett. 107 (141301) 2011, [arXiv:1106.0650]

[14] CDMS Collaboration, Dark Matter Search Results from the CDMS II Experiment, Science 26 (1619) March 2010

[15] CDMS Collaboration, Results from a Low-Energy Analysis of the CDMS II Ge Detectors, Phys. Rev. Lett. 106 (131302), 2011 [arXiv:1011.2482]

[16]CDMS Collaboration, Search for Annual Modulation in Low-energy CDMS II Data, [arXiv:1203.1309]

[17]EDELWEISS Collaboration, Final Results of the EDELWEISS-II WIMP Search Uing a 4-kg Array of Cryogenic Germanium Detectors with Interleaved Electrodes, Phys. Lett. B 702 (335) 2011 [arXiv:1103:4070]

[18]XENON100 Collaboration, Dark Matter Results from 100 Live Days of XENON100 Data, Phys. Rev. Lett. 107 (131302) 2011 [arXiv:1104.2549] 
[19] E. Aprile, XENON1T Collaboration, The XENON1T Dark Matter Search, in proceedings of DM2012 at UCLA [arXiv:1206.6288

[20]XMASS Collaboration, Scintillation-only Based Pulse Shape Discrimination for Nuclear Electron Recoils in Liquid XENON, [arXiv:1106:2209]

[21]A. Hime, The MiniCLEAN Dark Matter Experiment, in proceedings of DPF 2011 [arXiv:1110.1005]

[22]M.G. Boulay, DEAP Collaboration, DEAP-3600 Dark Matter Search at SNOLAB, in proceedings of TAUP 2011 [arXiv:1203.0604]. 